\title{
INSERCIÓN LABORAL DE LOS GRADUADOS DE INGENIERÍA COMERCIAL / ADMINISTRACIÓN DE EMPRESAS DE LAS UNIVERSIDADES CATEGORÍAS A Y B DE LA PROVINCIA DE PICHINCHA - ECUADOR
}

\author{
EMPLOYMENT INSERTION OF GRADUATES OF COMMERCIAL \\ ENGINEERING / BUSINESS ADMINISTRATION OF CATEGORY \\ A AND B UNIVERSITIES OF THE PROVINCE \\ OF PICHINCHA - ECUADOR
}

\author{
Rosario del Carmen Pineda López' Galo Ramiro Moreno Bastidas² \\ Roberto Xavier Manciati Alarcón ${ }^{3}$
}

\begin{abstract}
Resumen
El propósito de la investigación radica en identificar los factores que inciden en la inserción laboral de los graduados de la carrera de Ingeniería Comercial/Administración de Empresas de las Universidades categoría "A Y B" de la provincia de Pichincha, Ecuador. A nivel metodológico se analizará la situación actual de la inserción de los graduados, las limitaciones que estos han encontrado para su inserción laboral, las políticas de selección de personal de las empresas para determinar los perfiles y competencias requeridas, y finalmente, la opinión de los directores de las carreras sobre las acciones que las universidades encaran para facilitar la inserción de sus graduados. El presente estudio se concibe desde el enfoque mixto de la investigación, de tipo descriptivo con diseño de campo no experimental. Entre los principales resultados, el desarrollo de las principales competencias sobre las cuales se debe focalizar el esfuerzo son: capacidad para resolver problemas, trabajo en equipo, liderazgo, y toma de decisiones. Además, debe considerarse el dominio instrumental del idioma inglés. Para el desarrollo de las principales competencias serán necesarias actividades específicas orientadas a la resolución de situaciones concretas, mediante el análisis de casos de empresas o la presentación y evaluación de proyectos reales.
\end{abstract}

Palabras claves

Inserción laboral, empleabilidad, ingenieria comercial, administración de empresas., educación superior, egresados.

\begin{abstract}
The purpose of the research is to identify the factors that affect the labor insertion of graduates of the career of Commercial Engineering / Business Administration of the Universities category "A and B" of the Province of Pichincha Ecuador. Methodologically, the current situation of the insertion of graduates will be analyzed, the limitations that they have encountered for their labor insertion, the personnel selection policies of the companies to determine the profiles and skills required, and finally, the opinion of the Directors. of the careers on the actions that the universities undertake to facilitate the insertion of their graduates. The present study is conceived from the mixed approach of the investigation, of descriptive type with non-experimental field design. Among the main results, the development of The main competencies on which the effort should focus are: ability to solve problems, Teamwork, Leadership, and Decision-making. In addition, it should be considered that instrumental mastery of the English language. For the development of the main competences, specific activities will be necessary aimed at solving specific situations through the analysis of business cases or the presentation and evaluation of real projects.
\end{abstract}

\section{Keywords}

Job placement, employability, Commercial Engineering, Business Administration, Higher Education, Graduates.

$1 \quad$ Universidad de las Fuerzas Armadas ESPE, Quito - Ecuador (rcpineda@espe.edu.ec).

2 Universidad de las Fuerzas Armadas ESPE, Quito - Ecuador (grmoreno@espe.edu.ec)

3 Investigador Independiente, Quito - Ecuador (rmanciati@yahoo.com) 


\section{Introducción}

A nivel mundial, la empleabilidad de egresados universitarios tiene que enfrentar la realidad social y laboral de cada región en particular. Esta confrontación se debe al comportamiento asimétrico, económico y laboral global, por tanto, cada región tiene sus particularidades y las características de los egresados. El presente artículo de investigación aborda una problemática generalizada de la educación superior como lo es la inserción laboral de sus graduados y, además, se focaliza la investigación en dos carreras afines (Ingeniería Comercial y Administración de Empresas), que se dictan en la provincia de Pichincha, considerando que la oferta académica de esa región es significativa respecto de la general del Ecuador.

De acuerdo al reciente informe, Perspectivas sociales y del empleo en el mundo: tendencias 2019, de la OIT, el problema más agudo que enfrentan los mercados laborales del mundo es el empleo de muy baja calidad, esto significa que millones de trabajadores, incluso egresados universitarios, se ven en la obligación de soportar y asumir condiciones laborales deficientes (OIT/Cinterfor, 2019).

En el mencionado estudio se reporta que, en 2018, la gran parte de los 3300 millones de trabajadores en todo el mundo no disfrutaba de la seguridad económica que se espera de una relación laboral, tampoco tenían igualdad de oportunidades y mucho menos un bienestar generalizado. A pesar de la reducción del desempleo mundial, no se aprecia la mejora de la calidad del trabajo ni mucho menos la calidad de vida de los trabajadores (OIT, 2019).

Autores como Spicer (2017) sostienen que:

Los sistemas educativos de América Latina tienen dificultades para generar suficientes trabajadores calificados que contribuyan a aumentar la productividad. Más de un tercio de las empresas de la región citan el escaso nivel de capacitación de los empleados como una limitación importante. Para reforzar el crecimiento económico, América Latina debe invertir en una fuerza laboral competente. (p.7)

Ante estas evidencias observadas en el desequilibrio del mercado laboral, la conducción de las universidades de América Latina puede contribuir para que sus graduados adquieran las competencias necesarias y asi aprovechar las oportunidades que ofrece la región. Adicionalmente, el problema en el mercado laboral se agrava por una de las situaciones más difíciles en los últimos 15 años: la crisis económica global y el crecimiento paulatino de la población que han incidido directamente en la oferta y oportunidades de empleo (Álvarez y Romero, 2015; Solé, Sánchez, Arroyo y Argila, 2018).

En la última década, la inserción de los egresados universitarios al mercado laboral se ha convertido en un problema para las universidades, debido a su elevado crecimiento, lo que ha conllevado a realizar investigaciones sobre el destino de sus egresados. Ante esta situación, resulta necesario que los diferentes aspectos que forman parte de la realidad laboral de los graduados universitarios deban ser analizados, para orientar a los estudiantes y poner en marcha acciones que permitan optimizar la inserción laboral (García-García-Blanco, 2018; Simón, 2017).

En este orden de ideas, la situación actual del mercado laboral en Ecuador indica que, para el mes de junio de 2019, el desempleo nacional se ubicó en el $4.4 \%$, donde el desempleo urbano fue de 5.6 \% y el rural se ubicó en el $2.3 \%$. Estas mismas cifras, para el mismo período en el 2018 fueron: desempleo nacional $4.1 \%$; desempleo urbano $5.2 \%$ y el rural $2 \%$. Aun cuando se evidencia un leve aumento, estos no son significativos (INEC, 2019).

Por tanto, adquiere una significativa importancia investigar en la actualidad cómo se 
comporta la inserción laboral de los graduados de Ingeniería Comercial / Administración de Empresas de las Universidades categorías A y $B$ de la provincia de Pichincha, Ecuador.

\section{Planteamiento del problema y revisión bibliográfica}

\section{Planteamiento del problema}

La oficina de la Organización Internacional del Trabajo (OIT) para los países andinos (2018), informa que los problemas del mercado de trabajo para los jóvenes es un tema prioritario en las políticas públicas. La institución citada indica que en Ecuador, a finales del 2017, el porcentaje de jóvenes desempleados correspondía al $9.4 \%$ de la población joven en edad de trabajar. Del total de 338577 personas desempleadas a agosto 2018 , el $38.9 \%$ tiene una edad comprendida entre 15 y 24 años de edad, y de 1.6 millones de personas subempleadas en el país, el 20.9 \% tiene entre 15 y 24 años de edad.

La formación y los sistemas, sin embargo, serán condición necesaria mas no suficiente. A este respecto, Morueta, Tejeda y Cedeño (2015: 56), sostienen que "la inserción laboral de los nuevos profesionales demanda un monitoreo y seguimiento que permita determinar la empleabilidad en sus campos profesionales". En este contexto surgen las siguientes interrogantes de investigación:

¿Cuáles son los factores que determinan el éxito en la inserción laboral de los graduados de la carrera de Ingeniera Comercial/Administración de Empresas de las Universidades Categoría A y B de la provincia de Pichincha del Ecuador?, ¿reciben los egresados universitarios en las carreras objeto de estudio algún apoyo u orientación desde sus universidades de origen, para ubicarse en lo laboral, una vez culminados sus estudios?

Para dar respuestas a estas interrogantes se plantea el siguiente objetivo:
Identificar los factores que inciden en la inserción laboral de los graduados de la carrera de Ingeniería Comercial/Administración de Empresas de las Universidades categoría A y B de la provincia de Pichincha, Ecuador.

\section{Revisión bibliográfica}

\section{La inserción laboral}

Se entiende a la inserción laboral según Barrón y Rosas (2013) "como la consecución y desempeño de un empleo dentro de un área y sector acorde con la formación recibida" (p. 18). La inserción laboral constituye un observatorio de la generación de la desigualdad socioeconómica. Los estatus obtenidos en las primeras etapas de la vida laboral están cercanos a los factores de influencia, como el origen familiar, nivel educativo. Además, en las primeras etapas de la vida laboral se producen cambios de posición y con el análisis de esta movilidad se puede ver los efectos de la desigualdad de acceso al mercado de trabajo.

Otro término es la empleabilidad, que representa todos los elementos, conocimientos, competencia y habilidades profesionales y corrientes, constituidos de forma integral en un individuo como capacidad; de modo que la puesta en marcha promueve el beneficio económico y las expectativas del empleador (Carmona, 2002). Mientras que Pineda (2002) afirma que es el grupo de elementos que incrementan la factibilidad de que un sujeto halle, conserve y progrese en un trabajo.

\section{Situación actual de la inserción laboral de los egresados universitarios}

Feijó (2015), sostiene que, para entender cómo se puede insertar el profesional en el mercado laboral, determinar la relevancia de la administración de empresas en la matriz productiva del país en los patrones de desarrollo establecidos en la planificación nacional y encaminarse hacia las tendencias naciona- 
les e internacionales, es preciso conocer el desempeño empresarial en el país. Para Campos (2008), los vínculos entre la educación superior y el empleo pueden describirse como parte de un mérito educacional.

Rodríguez (2001), agrega que la inversión de tiempo, recursos financieros y energía que la sociedad, las familias y los propios individuos han hecho para alcanzar una titulación universitaria, se habrá indistintamente subutilizado si se trata de un individuo inactivo o de un desempleado. Pelayo (2012) agrega "El capital social es un elemento que está dentro de los factores de empleabilidad y comprende el contexto social de un individuo" (p. 16).

Jarvis (2012), plantea que los cambios en la economía, la inestabilidad y la competitividad, particulares del siglo XXI, han llevado a la exigencia del aprendizaje a lo largo de la vida, el cual se ha transformado en un referente para perfilar los currículos educativos y en una característica primordial imputada a las personas que tienen posibilidades de éxito en el ámbito profesional. La oferta educativa logra una nueva extensión en el aprendizaje, que amplía la visión tradicional de las etapas educativas orientadas a la formación profesional.

El aprendizaje permanente está asociado a un mercado de trabajo versátil, dinámico y que cambia de forma continua, en el cual debe permanecer la cualificación, la adquisición de nuevas competencias y la capacidad de cambiar a nuevas profesiones. Esto se vincula a profundas transformaciones sociales que exigen competencias personales para dar como resultado ciudadanos saludables, creativos, interactivos y comprometidos (Ministerio de Educación, 2011).

\section{Factores claves para acceder al empleo}

La mayoría de titulados universitarios consideran que entre los factores claves para acceder a un empleo se encuentran: personalidad, habilidades sociales, comunicación o capacidad de trabajar en grupo. De hecho, estos aspectos ligados a la personalidad y al modo de relacionarse con los demás parecen, en la mayoría de los casos, factores más relevantes para conseguir un empleo cualificado que los conocimientos propiamente dichos, como se refleja en el gráfico siguiente:

Figura 1. Factores importantes para conseguir trabajo de los graduados universitarios menores de 35 años que no están sobrecualificados

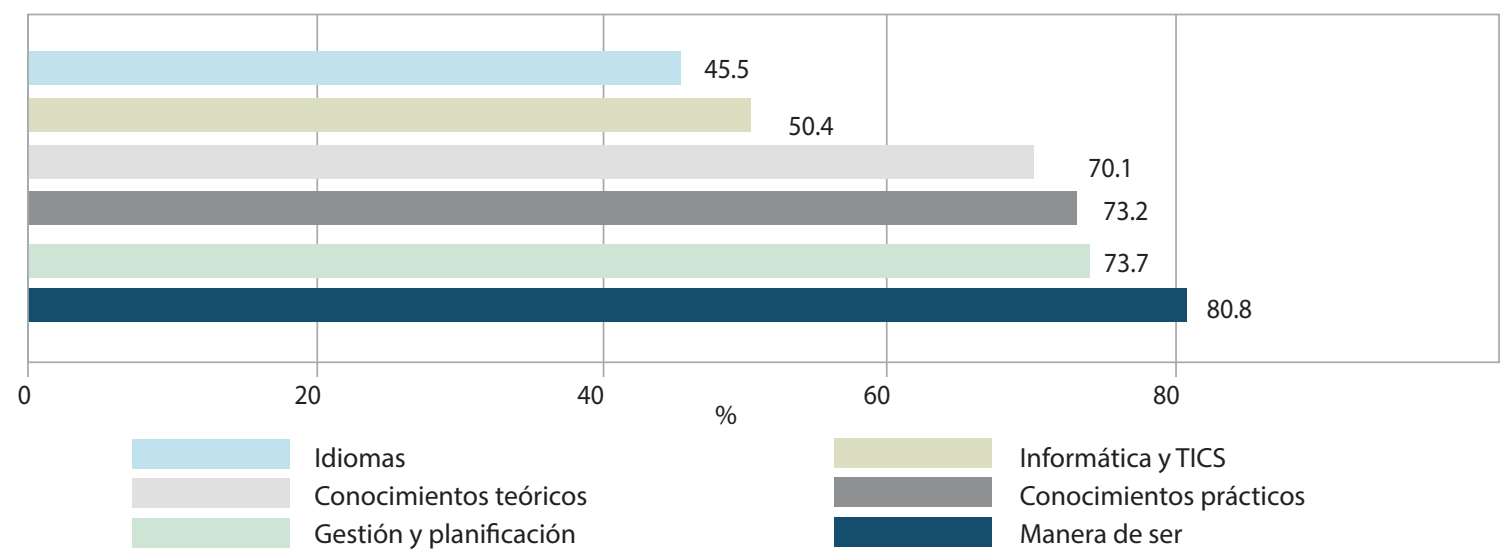

Las barras representan el porcentaje de titulados que afirman que ese factor fue bastante o muy importante para conseguir el trabajo actual.

Fuente: Encuesta de Inserción Laboral de Titulados Universitarios INE, 2014. $N=11.503$ 
Los datos mostrados en el gráfico proceden de la Encuesta de Inserción Laboral de Titulados Universitarios, elaborada por el Instituto Nacional de Estadística y Censos (INEC) en 2014. Se trata de un riguroso y exhaustivo estudio que contiene información de más de 30.000 universitarios que completaron una titulación en el curso 2009/2010 y fueron entrevistados 4 años después de salir de la Universidad. En los datos aquí mostrados, solo se considera a los titulados que en el momento de la entrevista se encontraban trabajando en un puesto adecuado a su formación, es decir, que no estaban sobre cualificados (Ramos, 2017).

\section{Metodología}

\section{Diseño de la Investigación}

El presente estudio se concibe desde el enfoque mixto de la investigación, a la vez es una investigación de tipo descriptiva (HernándezSampieri, Fernández-Collado, \& Baptista-Lucio, 2014), en tal sentido, procura estudiar y describir todos aquellos aspectos relacionados con la inserción laboral del grupo objeto de estudio. Se caracteriza por ser un estudio de campo, no experimental; así, recolectará toda la información directamente de los suje- tos investigados, en el sitio mismo de ocurrencia de los hechos, sin alterar ni modificar las variables de investigación, ni las condiciones existentes, de alli su carácter no experimental.

\section{Técnicas para la recolección de la información y su respectivo análisis}

Se aplicaron instrumentos debidamente validados tanto en confiabilidad como en contenido; este tipo de instrumentos fueron encuestas a los egresados universitarios, a los empleadores del sector empresarial y a los directivos universitarios encargados del seguimiento laboral de los egresados universitarios y de las bolsas de empleo.

Las variables a relacionar e investigar son: i) Variable directa: la empleabilidad de los egresados en Ingeniería Comercial/Administración de Empresas; ii) Variables indirectas: factores educativos, sociales y personales (socio-familiares).

\section{Población y muestra}

Las universidades de las categorías A y B de la provincia de Pichincha, las empresas y los graduados conformaron los 3 ejes de estudio de campo. De acuerdo a los ejes mencionados, la tabla 1 presenta la conformación de la muestra.

Tabla 1. Ejes del estudio de campo

\begin{tabular}{|c|l|l|l|}
\hline $\begin{array}{c}\text { Ejes de } \\
\text { estudio }\end{array}$ & \multicolumn{1}{|c|}{ Población } & \multicolumn{1}{c|}{ Muestra } & \multicolumn{1}{c|}{ Unidad de recolección } \\
\hline Universidades & $\begin{array}{l}\text { Directores delárea de inser- } \\
\text { ción laboral de las universi- } \\
\text { dades categorías A y B de la } \\
\text { provincia de Pichincha. }\end{array}$ & $\begin{array}{l}\text { Directores del área de in- } \\
\text { serción laboral de las uni- } \\
\text { versidades: EPN, ESPE, } \\
\text { PUCE, UCE, UPS y UTI. }\end{array}$ & $\begin{array}{l}\text { Directores de carrera y encargados } \\
\text { de seguimiento a graduados de las 6 } \\
\text { universidades en estudio: EPN, ESPE, } \\
\text { PUCE, UCE, UPS y UTI. }\end{array}$ \\
\hline \multirow{5}{*}{ Empresas } & $\begin{array}{l}\text { Empresas de la provincia } \\
\text { de Pichincha. }\end{array}$ & $\begin{array}{l}\text { Muestra representativa } \\
\text { de los directores o repre- } \\
\text { sentantes de las empre- } \\
\text { sas pequeñas, medianas } \\
\text { y grandes de la provincia } \\
\text { de Pichincha. }\end{array}$ & $\begin{array}{l}\text { Unidad de recolección: directores o } \\
\text { representantes de las empresas de la } \\
\text { provincia de Pichincha. } \\
\text { La población de estudio asciende a 19 } \\
\text { O75 directores o representantes, de } \\
\text { los cuales 377 representan la muestra } \\
\text { de estudio. }\end{array}$ \\
\hline
\end{tabular}




\begin{tabular}{|c|c|c|c|}
\hline Graduados & $\begin{array}{l}\text { Graduados de las univer- } \\
\text { sidades de las categorías } \\
\text { A y B de la provincia de } \\
\text { Pichincha, }\end{array}$ & $\begin{array}{l}\text { Muestra representativa } \\
\text { de los graduados de las } \\
\text { universidades EPN, ESPE, } \\
\text { PUCE, UCE, UPS y UTI. }\end{array}$ & $\begin{array}{l}\text { La población de estudio asciende a } \\
829 \text { graduados de las carreras de In- } \\
\text { geniería Comercial/Administración de } \\
\text { Empresas, en el año } 2016 \text { y de las } 6 \\
\text { universidades, de los cuales } 263 \text { re- } \\
\text { presentan la muestra de estudio. } \\
\text { EPN: } 7.10 \%, 19 \text { egresados } \\
\text { ESPE: } 11.10 \%, 9 \text { egresados } \\
\text { UCE: } 2.30 \%, 77 \text { egresados } \\
\text { PUCE: } 24.40 \%, 64 \text { egresados } \\
\text { UPS: } 22.40 \%, 59 \text { egresados } \\
\text { UTI: } 5.70 \%, 15 \text { egresados } \\
\text { TOTAL: } 263 \text { egresados }\end{array}$ \\
\hline
\end{tabular}

\section{Resultados y análisis}

\section{Situación de la inserción laboral de los graduados de las carreras de Ingeniería Comercial/Administración de Empresas - Universidades categoría "A y B" de Pichincha}

\section{Empleabilidad}

El porcentaje que está empleado es del $71.53 \%$, mientras que $22.99 \%$ no está empleado y un $5.5 \%$ nunca ha trabajado. Una estimación mediante intervalos de confianza para la población de graduados de Ingeniería Comercial/Administración de Empresas, que actualmente están con trabajo, es de: 66 $\% \leq \mathrm{P} \leq 77 \%$. De los que no tienen empleo, un 50 $\%$ ha estado desempleado por menos de 6 meses, un $23 \%$ menos de 1 año y un $26 \%$ más de un año.

\section{Trabajo relacionado a la profesión}

En relación al hecho de que los graduados trabajen actualmente en un ámbito relacionado a su profesión, un $55.48 \%$ de los encuestados muestreados tienen un trabajo relaciona- do con su profesión, donde un $16.1 \%$ considera que es muy relacionado con ella, el 44.52 \% asegura que su empleo está poco o nada relacionado con la carrera cursada, y el 11.31 \% opina que no está para nada relacionado.

Al inferir a la población se tiene:

- Trabajo relacionado a la profesión: 50 $\% \leq \mathrm{P} \leq 61 \%$

- Trabajo no relacionado a la profesión: $39 \% \leq \mathrm{P} \leq 50 \%$

Al analizar si la búsqueda de empleo ha tenido resultado en los graduados, un $49.64 \%$ de los encuestados manifestó haber buscado un empleo asociado a sus estudios académicos universitarios y a su vez haberlo encontrado, sin embargo, un porcentaje equivalente al $40.51 \%$, aun cuando se dedicó a la búsqueda, no pudo ubicarse en lo laboral. Un 9.85 \% nunca se dedicó a buscar empleo y colocarse en lo laboral.

Al analizar la realidad de empleo de los ingenieros comerciales / administradores, de acuerdo a la universidad en la que se graduaron, se puede observar lo siguiente:

Tabla 2. Empleo de acuerdo a la universidad

\begin{tabular}{|c|c|c|c|c|c|c|}
\hline Trabaja/Universidad & ESPE & PUCE & EPN & U. Central & UPS & UTI \\
\hline Sí & $77 \%$ & $81 \%$ & $82 \%$ & $57 \%$ & $63 \%$ & $60 \%$ \\
\hline No & $19 \%$ & $13 \%$ & $18 \%$ & $33 \%$ & $30 \%$ & $40 \%$ \\
\hline Nunca he trabajado & $4 \%$ & $6 \%$ & $0 \%$ & $10 \%$ & $7 \%$ & $0 \%$ \\
\hline
\end{tabular}


Los resultados muestran que las universidades ESPE, PUCE y EPN tienen porcentajes superiores a las de las universidades Central, UPS y UTI; es decir, tienen mayores niveles de empleabilidad.

\section{Tipo de empleo}

Al momento de la consulta efectuada, un $56.20 \%$ de los graduados manifestó estar empleado en relación de dependencia y el $23.36 \%$ se encontraba ejerciendo profesionalmente de manera independiente, lo cual indica que el $79.56 \%$ se encontraba trabajando. Por otra parte, se evidencia, con un 99 $\%$ de confianza, que las personas que trabajan en relación de dependencia tienen mayor satisfacción que las que laboran independientemente y para los desempleados mencionan una situación regular o mayormente mala.

\section{Empleabilidad en relación a género}

Al analizar si la empleabilidad depende del género, se observa mediante análisis Chi cuadrado, que la empleabilidad no depende del género, obteniéndose un valor $p=0.105$.

\section{Incidencia de la categoría \\ de la universidad de egreso \\ en la procura de un puesto de trabajo}

La investigación evidencia significativamente la predominancia de la universidad de donde egresó el Ingeniero Comercial/Administración de Empresas, para lograr una contratación laboral. Existe una opinión positiva al respecto, en un $64.96 \%$; no obstante, el restante $35.04 \%$ considera que no es así. Este resultado puede tener varias interpretaciones como: un sentido de pertenencia del graduado a su Universidad; o reconocimiento del sector empresarial sobre la calidad de la formación impartida por la categoría de la universidad, entre otras.

\section{Limitaciones en la inserción laboral de los graduados de las carreras de Ingeniería Comercial/ Administración de Empresas}

\section{Competencias y aptitudes \\ que tienen los graduados de Ingeniería Comercial/Administración de Empresas}

Para la valoración de las competencias y aptitudes se utilizó una escala de valor de 1 a 5 por cada una de las variables de análisis. El resultado promedio de los encuestados arrojó los siguientes resultados ${ }^{4}$. Al analizar las competencias que consideran los graduados tienen una vez que se gradúan de la universidad, se puede observar lo siguiente:

Las competencias con mayor frecuencia que los graduados mencionan que tenían cuando se graduaron son las siguientes: i) honestidad e integridad; ii) capacidad para el aprendizaje; iii) dedicación; iv) trabajo en equipo; v) relaciones interpersonales.

Mientras que habilidades como las siguientes, son las que no disponían mayormente en un inicio: i) conocimientos teóricos en su campo; ii) capacidad para aplicar los conocimientos en la práctica; iii) conocimiento de idiomas extranjeros; iv) criterio para la toma de decisiones; v) trabajo bajo presión; vi) capacidad para resolver problemas; vii) experiencia.

Sin descartar las demás, indican con una calificación mediana habilidades que no poseían como: i) tecnológica: buen conocimiento del uso de las TIC y aplicaciones informáticas; ii) pensamiento crítico ${ }^{5}$; iii) creatividad; iv) liderazgo; v) flexibilidad para adaptarse a los requerimientos del trabajo; vi) habilidades comunicativas.

\footnotetext{
$4 \quad$ Al ser un promedio de los 274 estudiantes, las variaciones pequeñas reflejan una diferencia importante, por lo que es necesario analizar uno a uno los resultados y valorar sus diferencias con este criterio.

$5 \quad$ Para profundizar los resultados ver anexo 6.5
} 
Por su parte, al analizar si poseen estas habilidades en la actualidad, el promedio es evidentemente mayor con un 3.8 en relación al promedio 3.21 que mencionaban cuando se graduaron, siendo un promedio mayor en todas las competencias mencionadas, mostrando que estas competencias y/o habilidades han incrementado en el tiempo y han aumentado, principalmente, habilidades como: dedicación, honestidad, capacidad para el aprendizaje, habilidades comunicativas. Finalmente, al analizar cuáles competencias consideran que les permitirían crecer, existe un puntaje bastante mayor en la respuesta, con un promedio de 4.12, indicando casi todas las competencias mencionadas como importantes para su crecimiento laboral.

Si se analiza de manera comparativa como importantes para crecer en el ámbito laboral, son las que tenían menor habilidad una vez que se graduaron: experiencia, capacidad para resolver problemas, criterio para la toma de decisiones, habilidades comunicativas, pensamiento crítico.

\section{Limitaciones cuando inician la relación laboral}

Los resultados en este sentido evidencian, de acuerdo a las respuestas de los Ingenieros Comerciales/Administración de Empresas, que al recibir su titulación experimentan dificultades al momento de ubicar e iniciar la primera relación de trabajo. La limitación de mayor frecuencia es la falta de experiencia, manifestada por el $43 \%$ de los profesionales, luego, el dominio de otro idioma con un $17 \%$, seguido de la remuneración aspirada versus la ofrecida por los empleadores con un $15 \%$. Las limitaciones que presentan menores porcentajes son el horario laboral (11\%), la formación académica (9\%), y el estado civil (5\%).

\section{Políticas de selección de personal en las empresas públicas y privadas de la provincia de Pichincha}

Caracterizando a las empresas objeto de estudio, estas se distribuyeron de la siguiente manera: $47.78 \%$ de las empresas pertenece al sector servicios, el $40.47 \%$ pertenece al sector comercial y el $\mathbf{1 1 . 7 5} \%$ pertenece al sector industrial.

El mayor porcentaje de empresas consideradas para el presente estudio pertenecen al sector privado con un porcentaje del $90.60 \%$, seguido por el sector público con un $7.31 \%$. Otros de menor importancia fueron la empresa mixta con un porcentaje equivalente al $1.83 \%$ y las Organizaciones no gubernamentales (ONG) con una participación relativa del $7.31 \%$.

En cuanto al número de empleados que trabajan en las empresas seleccionadas para la presente investigación, el $37.60 \%$ tiene entre 1 y 9 empleados; el $21.41 \%$ tiene entre 10 y 49 empleados; el $22.19 \%$ tiene entre 50 y 199 empleados y el $18.80 \%$ tiene más de 200 empleados.

\section{Entrevistas a directores de carrera - Participación de la universidad en la inserción laboral}

- La mayoría de las universidades tienen estudios sobre la inserción laboral de los graduados de la carrera de Ingeniería Comercial/Administración de Empresas, algunas recientes, otras lo actualizan periódicamente y algunas solo cuentan con esta información al momento del diseño original y puesta en marcha de los estudios de la carrera objeto de estudio. La mayoría cuenta con una unidad organizativa al respecto. Otras informan no tener información sobre los graduados y que 
solo se concentran en la información de los estudiantes regulares.

- La mayoría de las universidades evalúan los rediseños curriculares tomando en consideración estudios de mercado, entrevistas con el sector empresarial, contratando docentes actualizados en la disciplina objeto de estudio, y están dispuestos a considerar los hallazgos de la presente investigación.

- Sobre la existencia de la demanda de la carrera hay discrepancias y no se observa una prevalencia al respecto. Algunos consideran que sí existe demanda para la carrera pero que hay situaciones que afectan, como los altos costos universitarios o la situación económica del país; otros señalan que no hay una demanda muy satisfactoria por la probable saturación del mercado en virtud de que muchas universidades e institutos tecnológicos egresan profesionales afines a la Administración Comercial y por ello consideran la difícil inserción de los Ingenieros Comerciales/Administradores de empresas; otras universidades están realizando estudios al respecto para valorar la demanda y asumir decisiones al respecto.

\section{Conclusiones}

Entre las principales conclusiones obtenidas de la investigación se puede determinar.

\section{Inserción laboral desde el punto de vista de los graduados}

- Según la percepción de los graduados las competencias más importantes para crecer laboralmente son: experiencia, honestidad e integridad, criterio para la toma de decisiones, habilidades comunicativas, capacidad para resolver problemas, capacidad de aprendizaje, trabajo en equipo y pensamiento crítico.

- Según los graduados, la limitación de mayor frecuencia para el inicio de una relación laboral es la falta de experiencia, manifestada por el $43 \%$ de los profesionales; luego, el dominio de otro idioma con un $17 \%$, seguida de la remuneración aspirada versus la ofrecida por los empleadores con un $15 \%$. Finalmente, las limitaciones que presentan menores porcentajes son el horario laboral, la formación académica y el estado civil.

- La consulta permitió evidenciar que un $47.81 \%$ de los graduados vinculan de manera positiva la contribución de la universidad con la oportunidad de ubicar e iniciar una relación laboral satisfactoria, mientras que el 52.19 $\%$ menciona que no hubo una contribución directa de la universidad para conseguir empleo.

- Los resultados permiten inferir que el 62 \% tiene una opinión favorable generalizada sobre su formación universitaria. No obstante, la opinión de los graduados es que un título universitario no garantiza estatus social ni empleo pleno, por lo que es importante que se vincule la formación universitaria con el tipo de empleo requerido, para poder proporcionar los conocimientos y competencias que garanticen el éxito de la inserción al empleo del graduado universitario.

\section{Políticas de selección de personal d e acuerdo al punto de vista gerencial - empresarial}

- En el caso particular de la política de selección y contratación para cargos administrativos y afines, las respuestas de las empresas indican que el $27.94 \%$ no tiene preferencia por ningu- 
na universidad en particular, seguida de un $11.75 \%$ que prefiere los graduados de la Universidad Central, asimismo un $11.49 \%$ prefiere los graduados en Ingeniería Comercial de la ESPE.

- Los motivos empresariales para preferir a un graduado universitario procedente de determinada universidad evidencian que el $14.36 \%$ manifiesta la existencia previa de convenios con dichas universidades, igualmente el $13.84 \%$ tiene como base los niveles de competencia y conocimientos alcanzados por los graduados, mientras que un $8.61 \%$ lo hace por la experiencia de sus graduados.

- Para las empresas, las competencias más requeridas al momento de contratar personal son: flexibilidad, capacidad para resolver problemas, trabajo en equipo, liderazgo, toma de decisiones, honestidad, experiencia y trabajo bajo presión.

- Al contrastar estas competencias con las que mencionan los graduados como las más importantes, se observa una coincidencia importante, especialmente en lo referente a: capacidad para resolver problemas, trabajo en equipo, honestidad, toma de decisiones, experiencia.

- El sector empresarial consultado cree que deben mejorarse, en el ámbito de la formación universitaria de los Ingenieros Comerciales y Administradores de Empresas, las siguientes competencias por orden de importancia: innovación, ética profesional, uso de TIC, mejora continua, actitud, aplicación de modelos empresariales e idiomas.

\section{Bibliografía}

Álvarez, J. y Romero, A. (2015). La empleabilidad de graduados universitarios en el contexto latinoamericano. Realidades de Unian- des, Ecuador. Atenas 32, Vol. 4 (octubre diciembre). Recuperado de: https://Www. redalyc.org/pdf/4780/478047208001. pdf, pp. 01-15.

Barrón, C. y Rosas, M. J. (2013). Seguimiento de egresados de doctorado en Pedagogía de la UNAM. Recuperado de www.amet.uady. $\mathrm{mx} /$ ? dl_name $=$ CONCEPCIN_BARRIN_TIRADO_JESICA_MARTINEZ_ROSAS.PDF

Campos, M. (2008). Los egresados y su inserción laboral ¿estudias o trabajas? . Revista Fuentes, 8.

Carmona, G. (2002). El Plan Personal para la Inserción socio-laboral. Teoría y práctica en la empresa. Huelva: Universidad de Huelva y Asociación de Empresas de Inserción de Andalucía.

Feijó, N. (2015). Seguimiento a graduados: inserción laboral y mejoramiento continuo de la escuela de Administración de empresas. RevistaECA Sinergia, 7, 45.

Feijó-Cuenca, N., Moreira-Zambrano, J. y FeijóCuenca, T. (2015). Seguimiento a graduados: Inserción laboral y mejoramiento continuo de la Escuela de Administración de Empresas. Revista ECA Sinergia. ISSN 1390-6623. FCAE. U.T.M. Dic, Año 6 Vol. 7, p. 44. Recuperado de: https://dialnet.unirioja.es/servlet/articulo?codigo $=6197623$, pp. 44-57.

García, J. y Pérez, M. (2008). Espacio Europeo de Educación Superior, competencias profesionales y empleabilidad. Revista Iberoamericana de Educación, 9(46).

García-Blanco, M. y S. (2018). La inserción laboral en la Educación Superior: Perspectiva:La perspectiva Latinoamricana. Educación , $323-347$.

Hernández-Sampieri, R., Fernández-Collado, C. y Baptista-Lucio, M. (2014). Metodología de la investigación Sexta Edición. México, D.F.: McGraw-Hill / Interamericana Editores, S.A. de C.V.

INEC. (2019). Encuesta nacional de empleo, desempleo y subempleo (ENEMDU). Quito: Ecuador en cifras. En línea: https://www. ecuadorencifras.gob.ec/documentos/ web-inec/EMPLEO/2019/Junio/Boletin. tecnico_de_empleo_jun19.pdf. 
Jarvis, P. (2012). Globalisation, Lifelong Learning and the Learning Society. (0. Routledge., Ed.) Sociological perspectives: Lifelong learning and the Learning Society, 2.

Ministerio de Educación. (2011). Plan director de empleabilidad universitaria.

Morueta, R., Tejeda, R. y Cedeño, G. (2015). Implementación institucional de un modelo cooperativo para el seguimiento a graduados en Ecuador. Rev Educ Sup , 125-56.

Oficina de la OIT para los Países Andinos. (2018). ECUADOR: Programa de acción 20182019. Quito: En línea: https://www.ilo.org/ wcmsp5/groups/public/---americas/- -ro-lima/documents/publication/ wcms_644921.pdf.

OIT. (13 de febrero de 2019). Perspectivas sociales y del empleo en el mundo - Tendencias 2019. Obtenido de El gran problema del empleo en el mundo: Las malas condiciones de trabajo: https://www.ilo.org/ global/about-the-ilo/newsroom/news/ WCMS_670577/lang--es/index.htm

OlT/Cinterfor. (2019). Empleabilidad. Recuperado de Cinterfor: http://www.oitcinterfor. org/?q=taxonomy/term/3406
Pelayo, M. B. (2012). Capital social y competencias profesionales: Factores condicionantes para la inserción laboral. eumed.net.

Pineda, P. (2002). Pedagogía laboral. Barcelona: Editorial Ariel.

Ramos, M. (2017). Las habilidades no cognitivas y la universidad. Blog Universidad. Recuperado de http://www.Universidadsi.es/lashabilidades-no-cognitivas-la-Universidad/

Rodríguez, C. (2001). La inserción laboral de egresados de la educación superior en el estado de Hidalgo. Centro de Investigación en Ciencias y Desarrollo de la Educación. Recuperado de: http://publicaciones.anuies. $m x / p d f s / r e v i s t a / R e v i s t a 127 \_S 2 A 1 E S$. $p d f(127)$, pp.7-22. .

Romero, V., Pérez, E., Vidal, S. y Juez, M. (2013). Inserción ocupacional. Barcelona: Altamar.

Simón, J. (2017). Egresados de nuevas carreras universitarias: Competir desigualmente en el mercado de trabajo. Revista de psicología educativa, 1-27.

Solé, M., Sánchez, J., Arroyo, F. y Argila, A. (2018). Los egresados universitarios y la inserción laboral: un acercamiento al panorama latinoamericano y español. Revista CEA, 67-74.

Spicer, D. (2017). Action learning in an MBA program. Education+ Training, 7-54. 